\title{
GERONTOPSICOMOTRICIDADE E OS EFEITOS DA ATIVIDADE FÍSICA SOBRE O DECLÍNIO COGNITIVO DECORRENTE DA DOENÇA DE ALZHEIMER
}

\author{
GERONTOPSYCOMOTRICITY AND THE EFFECTS OF PHYSICAL ACTIVITY ON COGNITIVE \\ DECLINE RESULTING FROM ALZHEIMER'S DISEASE
}

\author{
Rafael Soares Silva \\ Universidade Federal Rural do Rio de Janeiro, Rio de Janeiro, RJ, Brasil. E-mail: doc.rafaelsoares@ \\ gmail.com
}

Fabio José Antonio da Silva

Universidade Estadual de Londrina, Londrina, PR, Brasil. E-mail: fjas81@hotmail.com

DOI: https://doi.org/10.46550/amormundi.v2i9.143

Recebido em: 11.12.2021

Aceito em: 27.12.2021

\begin{abstract}
Resumo: A gerontopsicomotricidade é uma área da psicomotricidade que se dedica ao estudo de intervenções psicomotoras para indivíduos idosos, visando uma boa relação entre habilidades motoras, cognitivas, sociais, psicológicas e afetivas. Com o aumento da população de idosos e da expectativa de vida, destaca-se a importância de as pessoas envelhecerem de forma ativa e saudável. Entre as principais doenças que acometem estes sujeitos, destaca-se a doença de Alzheimer (DA), uma enfermidade neurodegenerativa que causa a perda progressiva de memórias recentes e prejuízos neuromotores. Posto isto, o presente trabalho elencou através de uma revisão indícios sobre a importância da atividade física regular no tratamento e prevenção da DA.
\end{abstract}

Palavras-chave: Gerontopsicomotricidade. Doença de Alzheimer. Atividade física. Tratamento. Prevenção.

Abstract: Gerontopsychomotricity is an area of psychomotricity that is dedicated to the study of psychomotor interventions for elderly individuals, aiming at a good relation between motor, cognitive, social, psychological and affective abilities. With the increase in the population of the elderly and the life expectancy, the importance of people aging in an active and healthy way stands out. Among the main diseases that affect these subjects, stands out Alzheimer's disease (AD), a neurodegenerative disease that causes progressive loss of recent memories and neuromotor damage. Therefore, the present study lists through a review clues about the importance of regular physical activity in the treatment and prevention of $\mathrm{AD}$.

Keywords: Gerontopsychomotricity. Alzheimer's disease. Physical activity. Treatment. Prevention. 


\section{Introduçáo}

$\Lambda$ evoluçáo das ciências médicas, tecnologias e políticas públicas dedicadas ao campo da

- saúde influenciaram drasticamente na expectativa de vida da população. De acordo com o Instituto Brasileiro de Geografia e Estatística (IBGE), entre o período de 1940 até 2016, a longevidade da população brasileira aumentou em média 30 anos. Atualmente, a expectativa média de vida no Brasil é de 75 anos (HOSPITAL SÃO CAMILO et al., 2018).

Não há dúvida de que houve um crescimento exponencial da população de idosos no mundo de forma geral, sendo assim, doenças relacionadas à idade aumentam dramaticamente. Um dos distúrbios mais comuns relacionados à idade avançada é a demência, sendo a doença de Alzheimer (DA) a causa mais comum e que vem sendo estudada a partir de várias áreas do conhecimento (biomarcadores, estrutura cerebral, comportamento e comprometimento cognitivo).

Para envelhecer de forma ativa e saudável recomenda-se uma série de hábitos saudáveis envolvendo uma alimentação equilibrada, redução do consumo de bebidas alcoólicas e tabaco, diminuição do estresse psicológico - evitando situações estressantes ou até mesmo através de tratamento psicoterapêutico, realizar consultas médicas periódicas e praticar exercícios físicos regularmente.

É do conhecimento comum que a atividade física regular deva fazer parte de um estilo de vida saudável em todas as faixas etárias. Os benefícios de saúde relacionados ao exercício físico são muitos, até mesmo durante a terceira idade. O potencial efeito positivo da atividade física sobre a cognição de pessoas idosas tornou-se um foco oportuno na literatura, dando outro significado ao "envelhecimento" (DORNELLES, 2003).

Diversos estudos têm mostrado que a atividade física regular traz melhorarias significativas para a saúde e qualidade de vida em indivíduos idosos, além de diminuir ou impedir o declínio funcional associado ao envelhecimento. Com relação à DA, muitas pesquisas relatam que o exercício físico está associado a taxas mais baixas de rebaixo cognitivo típicos dessa enfermidade.

Ao longo do tempo, houve muito esforço da comunidade científica para identificar precocemente indivíduos que estão em risco para DA. Vários estudos visam testar intervençóes com objetivo de atrasar a progressão dos sintomas para a demência completa. Os resultados de pesquisas científicas observacionais sugerem que pessoas idosas que demonstram freqüentemente perda progressiva de memória ou evidenciam algum tipo de comprometimento cognitivo, estão mais propensos a desenvolver Alzheimer futuramente. A prática de atividade física regular está associada à redução da incidência de demência em ambos os sexos (POIRIER; GAUTHIER, 2016).

A partir destes pressupostos teóricos, o presente trabalho, de cunho bibliográfico, teve como objetivo ressaltar a importância da gerontopsicomotricidade e revisar na literatura científica pesquisas significativas que evidenciam contribuiçôes da prática de atividade física regular sobre o declínio cognitivo de indivíduos idosos diagnosticados com DA. É magnífico o fato das pessoas terem a possibilidade de uma vida longa, porém, certos cuidados para que a longevidade aconteça de forma natural e saudável são necessários, principalmente em relação ao desenvolvimento neurológico, sendo assim, destaca-se a importância desta pesquisa, uma vez que a mesma esclarece de forma clara e objetiva os benefícios da realização de exercícios físicos 
regulares na redução dos sintomas cognitivos típicos da DA e na prevenção desta enfermidade.

\section{Visáo geral sobre a doença de Azheimer}

A primeira descrição oficial de DA aconteceu em 1906, por um importante psiquiatra alemão da época, chamado Alois Alzheimer, o qual teve seu feito homenageado no nome da doença, dando origem ao termo "doença de Azheimer". O primeiro caso descrito foi de uma mulher que apresentava um conjunto de sintomas relacionados à perda de memória, deterioração de habilidades cotidianas e alteraçôes comportamentais. Após a morte desta paciente, Dr. Azheimer realizou estudos post-mortem com o sistema nervoso central da mesma, identificando alteraçôes neuroanatômicas incomuns (LOPES; BOTTINO, 2002).

A DA é caracterizada como uma doença neurodegenerativa e progressiva, a qual causa perda gradativa de memórias recentes e rebaixamento do funcionamento cognitivo. Geralmente, os primeiros sintomas ocorrem no final da vida adulta. Ainda não existe um consenso na comunidade científica sobre a etiologia dessa enfermidade. Achados neuropatológicos apontam como possível causa o alto acúmulo intracelular de proteína amilóide. No entanto, nem todos os indivíduos que apresentam essa condição neuropatológica prototípica manifestam os sintomas clínicos de demência.

Dentre os fatores de risco destaca-se o histórico familiar, nível de escolaridade, engajamento social, desenvolvimento cognitivo e traumatismo cranioencefálico. No entanto, o fator risco principal é a idade avançada.

A heterogeneidade dos sintomas é muito ampla, mas a manifestação comum entre todos os casos é a incapacidade insidiosa em se lembrar de informaçóes recentes. À medida que neurônios em diversas partes do sistema nervoso central apresentam baixo funcionamento e morrem, outros sintomas se desenvolvem, incluindo uma perda grave de memória que pode afetar as atividades da vida diária, dificuldades na solução de problemas, desorientaçáo, falta de noção visual-espacial e complicaçóes psicomotoras progressivas. Eventualmente, podem ocorrer problemas neuropsiquiátricos, tais como alterações de humor e personalidade (CAIXETA, 2009).

Conforme a progressão da doença é comum a manifestação de sintomas cognitivos, incluindo dificuldades psicomotoras progressivas,

O diagnóstico em sua maior parte é clínico e envolve a obtenção de um bom histórico que inclua informações médicas, familiares e neuropsiquiátricas. Exames laboratoriais são essenciais para descartar outras causas de demência (hemograma completo, amplo painel metabólico, hormônio estimulante da tireóide, vitamina B12 e ácido fólico), bem como exames de neuroimagens com ressonância magnética (RM). O diagnóstico pode ser reforçado com testes neuropsicológicos e sorologias para biomarcadores, conforme necessário. Os biomarcadores atualmente podem ser obtidos do sangue ou do líquido cefalorraquidiano. Os principais biomarcadores do líquido cefalorraquidiano apresentam alta precisão diagnóstica, com sensibilidade e especificidade de $85 \%$ a $90 \%$.

O tratamento farmacológico atual envolve inibidores da acetilcolinesterase e antagonistas do receptor $\mathrm{N}$-metil $\mathrm{D}$-aspartato (memantina), os quais contribuem minimamente nos estágios iniciais da doença, retardando a progressão da DA em estágios posteriores e fornecem algum 
alívio sintomático, mas não alcançam uma cura definitiva. Por estas razões, o exercício físico tem sido explorado como um meio viável de prevenção e tratamento para doença em estágio inicial e tardio, devido à sua relativa segurança e poucos efeitos colaterais (CARPER, 2015).

\section{Conceitualização de gerontopsicomotricidade}

Antes de conceituar a gerontopsicomotricidade é preciso entender o significado do conceito de psicomotricidade, uma vez que a primeira é uma subdivisão da segunda. A psicomotricidade é compreendida como uma ciência que se dedica aos estudos pertinentes da relação entre o corpo e a mente. Nesse sentido, o ser humano é visto como um todo: movimentos corporais, sentimentos, pensamentos, emoçóes e contexto social.

As intervençôes psicomotoras visam compreender uma pessoa em vários de seus aspectos, incluindo as capacidades motoras, psicoemocionais, cognitivas e sociais - tudo isso para elaborar o plano de intervenção mais ideal para cada caso. Estas intervençôes podem ser lúdico-terapêuticas, exercícios expressivos, relaxação e atividades motoras.

A gerontopsicomotricidade, por sua vez, é exclusivamente dedicada aos indivíduos da terceira idade, permitindo ao geronte ou idoso desenvolver habilidades psicomotoras de forma terapêutica e preventiva através de terapias não farmacológicas, as quais estudam o movimento corporal de pacientes idosos e sua relação com aspectos psicológicos e afetivos.

O psicomotricista que atua na área da gerontopsicomotricidade trabalha com objetivo de neutralizar ou minimizar a retrogénese psicomotora, a diminuição das capacidades cognitivas, a perda de capacidades sensoriais, os problemas emocionais e afetivos. Essa ciência cada vez mais vem ganhando importância ao longo do tempo, uma vez que a mesma de forma simples eleva a importância de valores psicoafetivos por meio de atividades lúdicas e interativas, permitindo um envelhecimento ativo e saudável.

É importante ressaltar que a gerontopsicomotricidade desempenha um papel importante no retardo dos sintomas de determinadas doenças, incluindo mal de Parkson, síndrome pósqueda, acidente vascular cerebral, depressão, ansiedade, problemas na motricidade (marcha, coordenação, equilíbrio e ocorrência de quedas), doença de Alzheimer etc.

As intervençôes psicomotoras em pessoas idosas podem ser classificadas em primárias, secundárias e terciárias. As primárias visam prevenir possíveis doenças que surgem ao longo do envelhecimento. As secundárias têm como principal característica a reabilitação, atuando em sujeitos ligeiramente problemáticos a nível cognitivo e/ou funcional, desenvolvendo as capacidades ainda preservadas e estimulando as que estão em processo de deterioração. As terciárias ocorrem nos casos em que já existe um diagnóstico estabelecido, nos quais os sintomas cognitivos e funcionais estão mais evidentes, visando o retardo dos mesmos e ao mesmo tempo promover a autonomia em atividades cotidianas e a qualidade de vida (FONSECA, 1988).

4 Atividade física regular como proposta de intervençáo: visando atrasar ou minimizar o declínio cognitivo em pessoas idosas diagnosticadas com a doença de Alzheimer

Um tratamento terapêutico para a DA deve estar focado em melhorar a qualidade de vida, detendo o declínio mental e promovendo a auto-estima do paciente. Além disso, as 
atividades terapêuticas para essa populaçáo precisam proporcionar prazer imediato, restabelecer a dignidade humana, fornecer tarefas significativas, restaurar papéis do cotidiano e possibilitar o convívio social (MARSHALL; HUTCHINSON, 2001).

Dado o potencial de efeitos colaterais dos medicamentos, tem havido um grande interesse no tratamento não farmacológico (STRÖHLE, et al., 2015). Tratamentos como o treinamento cognitivo e estimulação cognitiva, demonstraram benefícios mistos e inconsistentes (SZETO; LEWIS, 2016). O exercício físico regular, por sua vez, mostrou-se promissor na prevenção e no tratamento de DA e outros tipos de demência.

Pessoas idosas com vários níveis de comprometimento cognitivo podem se beneficiar de exercícios multimodais, sistematizados e supervisionados, quando praticados num intervalo de aproximadamente 60 minutos por dia, de 2 a 3 dias por semana, para melhorar a funçóes físicas e psíquicas. $\mathrm{O}$ exercício físico melhora a força, equilíbrio, mobilidade e resistência em pessoas com comprometimento cognitivo e demência (LAM et al., 2018).

Em 2006, na Universidade Paulista (UNESP) de Rio Claro foi criado um projeto de extensão do Departamento de Educação Física, denominado de "Programa de Cinesioterapia Funcional e Cognitiva em Idosos com Doença de Alzheimer" (PRO-CDA). O objetivo deste projeto é de propiciar aos pacientes participantes os benefícios obtidos através da prática regular de atividade física para a melhoria dos sintomas progressivos da DA, visando à qualidade de vida deles e de seus cuidadores. A equipe que comanda o programa é composta por profissionais das áreas da Educação Física, Medicina, Fisioterapia, Psicologia, Psiquiatria, além de contar com estagiários graduandos dos cursos de Educação Física e Fisioterapia (GARUFFI et al., 2011).

Uma amostra de 16 idosos diagnosticados com DA, sendo que sete pertenciam ao grupo controle, foram submetidos a um estudo com objetivo de analisar os efeitos de um programa composto por diferentes tipos de atividades físicas regulares, sistematizadas e supervisionadas sobre as funçóes executivas, risco de queda e equilíbrio. As intervençóes aconteceram três vezes por semana, em um período de seis meses. Cada sessão durou aproximadamente 60 minutos, as quais visaram à estimulação motora e cognitiva, simultaneamente ou isoladamente, incluindo atividades de alongamento, dança, relaxamento, ludicidade, circuitos, prática de esportes e treinamento com pesos. A partir dos resultados, os pesquisadores concluíram que a corporeidade exercida regularmente de forma sistemática e supervisionada é uma ótima abordagem nãofarmacológica, beneficiando as funçôes cognitivas, equilíbrio e risco de quedas. Além do mais, foi evidenciado que a agilidade e o equilíbrio estão relacionados com funçóes executivas em idosos com Azheimer (HERNANDEZ et al., 2010).

Diversas pesquisas realizadas nas últimas décadas sugerem que num futuro próximo a prevenção da DA será baseada em regras que regem os hábitos de vida, incluindo uma dieta saudável, estímulos cognitivos e exercícios físicos. Vários fatores, em especial a atividade física, podem contribuir na prevenção do declínio cognitivo e retardar o início da demência, ou seja, uma vida ativa confere um efeito protetor sobre o funcionamento cerebral dos idosos, retardando o curso dos sintomas (ROLLAND; KAN; VELLAS, 2008).

[...] a prática regular de atividade física sistematizada, preferencialmente associada à estimulaçấo cognitiva, contribui para a preservação ou mesmo melhora temporária de várias funçôes cognitivas, particularmente de atenção, funçôes executivas e linguagem, em pacientes com DA. Diante da relevância do tema 
e dos desafios ao tratamento desta doença, a prática regular de atividade física sistematizada representa uma intervenção nãofarmacológica benéfica para estes pacientes. Há a necessidade de novos estudos controlados para se aferir quais procedimentos concomitantes de intervenção motora e de estimulação cognitiva seriam mais apropriados como recursos adicionais ao tratamento na DA. (COELHO et al., 2009, p. 6).

A literatura epidemiológica fornece evidências convincentes de que maiores quantidades de atividade física estão associadas ao risco reduzido de demência na terceira idade. Além disso, intervençóes randomizadas utilizando ferramentas de neuroimagem relataram que a prática de atividade física está associada ao aumento de atividade neurológica em diversas regiôes do sistema nervoso central, incluindo o cortex pré-frontal e o hipocampo, o que pode levar a uma reduçáo nos prejuízos da memória. Estudos longitudinais utilizando ferramentas de neuroimagem também mostram que o volume de áreas cerebrais pré-frontais e hipocampais é maior em indivíduos que se engajaram em atividade física precocemente durante a vida.

O fluxo sangüíneo cerebral é adversamente afetado com o avanço da idade e está associado à cognição. $\mathrm{O}$ exercício físico regular, mesmo que praticado em intensidade moderada, demonstra resultados significativos no aumento do fluxo sanguíneo no sistema nervoso central (BARNES; YAFFE, 2011). Um estudo identificou maiores níveis de circulação sangüínea no cérebro de homens empenhados em atividades físicas regulares quando comparado com homens sedentários (BAILEY et al., 2013). Comparaçôes de exames de ressonância magnética e medidas fisiológicas, realizados em idosos, antes e depois da prática de exercícios aeróbicos, mostram um aumento do fluxo sanguíneo cerebral em regiôes neurológicas associadas à função executiva e memória (KUSTER et al., 2016).

A atividade física em um ambiente favorável estimula o cérebro em um nível corporal e cognitivo e é benéfica para a plasticidade cerebral (capacidade neuronal de mudar e adaptarse como resultado da experiência) em pacientes com demência. Embora a demência seja uma doença incurável, sua progressão pode ser retardada através de múltiplas atividades (ZENG et al., 2016).

Exercitar o corpo de forma sistemática promove a neuroplasticidade, principalmente quando se trata de atividades que envolvem a combinação de habilidades mentais e físicas, associadas aos circuitos cerebrais cognitivo-associativos. Quando a atividade física é incrementada com estímulos cognitivos de maneira controlada, a auto-organização e a conectividade entre os sistemas cerebrais aumentam. Tal intervenção tem demonstrado benefícios significativos em idosos diagnosticados com DA em habilidades pertinentes à função executiva (GUERRA et al., 2017).

Os benefícios de atividades físicas realizadas regularmente estão relacionados à maximização da função cognitiva, logo, cada vez mais esta prática é reconhecida por promover a saúde mental e como um grande potencial no combate a DA, oferecendo uma intervenção de baixo custo e baixo risco e que está amplamente disponível, podendo ter efeitos transformadores no quadro sintomático. Demonstrar que o engajamento em atividade física altera o curso dos sintomas, tem enormes implicaçóes na saúde pública das pessoas idosas (VIDONI et al., 2012).

Sobol e sua equipe ministraram um estudo no qual participaram 200 idosos com Alzheimer, sendo a idade média da amostra de 71 anos. $\mathrm{O}$ objetivo foi de identificar os diversos benefícios dos exercícios aeróbicos sistematizados em pessoas idosas quanto à progressão dos 
sintomas da doença. Cada sessão durou aproximadamente uma hora, ocorrendo três vezes por semana em um período de três meses. Após todas as sessóes, testes clínicos identificaram avanços na aptidão cardiorrespiratória, desempenho físico em tarefas cotidianas e melhorias na realização de tarefas simultâneas (SOBOL et al., 2016).

Um determinado estudo foi realizado com objetivo de investigar os benefícios de exercícios aeróbicos para a melhoria de habilidades da função executiva em uma amostra de 28 idosos com diagnóstico para DA, sendo a média de idade entre os mesmos foi de 78 anos. Através de testes psicológicos e entrevistas com os pacientes, conclui-se que a atividade aeróbica praticada regularmente é uma intervenção eficaz na manutenção da função executiva dessa população, além do mais, evidenciou-se uma redução significativa no declínio da cognição global (YU; VOCK; BARCLAY, 2018).

Um grupo de pesquisadores desenvolveu um estudo com objetivo de identificar possíveis melhorias na vida de pessoas diagnosticas com algum tipo de demência - incluindo a DA, através de exercícios físicos proporcionados pela locomoção em bicicletas. A amostra estudada foi composta por 51 indivíduos idosos, os quais pedalaram por 15 minutos diários durante um período de 15 meses. Por meio de testes clínicos percebeu-se que houve avanços significativos na função executiva, melhorias nos sintomas neuropsiquiátricos, memória e mobilidade funcional. Outro aspecto importante desta pesquisa foi de que os exercícios praticados conferiram maior efeito naqueles sujeitos que tinham um quadro mais grave de comprometimento cognitivo (CANCELA et al., 2016).

Alguns pesquisadores atribuem outros benefícios para atividades físicas, incluindo alteraçóes positivas na neurogênese e a formação de novas redes neurais (SHEN; LI, 2016).

A neurogênese do hipocampo em adultos é crucial para o aprendizado e memória (RYAN; NOLAN, 2016). O hipocampo é reconhocido como uma região nerológica de extrema importancia para um envelhecimento saudável, pois o mesmo é fortemente relacionado com memórias de curto e longo prazo. Portanto, há evidências convincentes de que a atividade física tem uma relação importante com regiôes cerebrais implicadas no declínio cognitivo, as quais estão associadas ao avanço da idade e aos sintomas do Alzheimer (ERICKSON; WEINSTEIN; LOPEZ, 2012).

Circuitos hipocampais também são considerados muito importantes para a memória episódica, os quais são afetados na DA durante a fase inicial dos sintomas. Acredita-se que grandes volumes hipocampais estejam associados a um melhor funcionamento cognitivo. A prática de exercícios leves e moderados apontam para uma possível prevenção de atrofia no volume do hipocampo. Além disso, o desenvolvimento hipocampal também está correlacionado com as alteraçóes da aptidão cardiovascular (DUZEL; PRAAG; SENDTNER, 2016).

Lourenço et al. (2019) ministraram um estudo com objetivo de estabelecer uma relação entre o hormônio irisina e atividade física com a DA, levando em consideração a plasticidade cerebral e a memória, utilizando humanos e modelos experimentais. No corpo humano, a síntese de irisina é estimulada pela prática de exercícios físicos. Este é hormônio é detectado em quantidades reduzidas no liquido cefalorraquidiano e no hipocampo de idosos com DA e em camundongos sedentários. A irisina participa ativamente na estimulaçáo de uma proteína muito importante ao funcionamento do hipocampo, a BDNF. Desse modo, conclui-se que irisina atua como fator de neuroproteção, influenciando a função executiva. 
Quando um vetor que expressa produção de irisina no cérebro é injetado via intravenosa em camundongos, após certo tempo, detecta-se um aumento no córtex e no hipocampo, sugerindo que administrar doses periféricas do vetor faz com que o mesmo atinja o sistema nervoso central e desencadeie o aumento exponencial de irisina, sendo assim, parecendo uma estratégia formidável naqueles casos em que praticar atividade física seja algo inviável, ressaltando a importância desse hormônio para um possível tratamento farmacológico ou induzido em pacientes com DA.

\section{Consideraçóes finais}

A gerontopsicomotricidade é uma área muito importante da psicomotricidade, a qual trabalha de forma terapêutica e preventiva na população idosa. Apesar do caráter predominantemente preventivo desta ciência, ela também pode atuar na reabilitação de gerontes com demência, principalmente quando se trata da DA. Partindo desse pressuposto, conclui-se que a gerontopsicomotricidade é competente em estimular sujeitos idosos para a descoberta de seus próprios corpos, ou seja, os motivando para realizaçáo de diferentes atividades, promovendo a manutenção e/ou recuperação da autonomia, além de desenvolver neles a capacidade relacional.

A partir desta pesquisa, foi possível perceber que o envelhecimento humano é um processo contínuo e gradual, contemplando alteraçóes naturais que se intensificam no final da fase adulta - a terceira idade. O organismo com o passar da idade sofre mudanças que são normais, esperadas e inevitáveis, principalmente em relação ao declínio cognitivo. No entanto, alguns idosos náo envelhecem cognitivamente dentro da normalidade, porque são mais propensos a desenvolverem quadros clínicos de demência, levando-os a uma perda acelerada da capacidade cognitiva, afetando diversas áreas do funcionamento neurológico, incluindo a memória de curto prazo, função executiva, noção visual-espacial, etc.

Profissionais da área da Educação Física em parceria com profissionais da Medicina, não devem medir esforços a fim de buscar intervençóes promissoras e no desenvolvimento de pesquisas visando um envelhecimento ativo e saudável. Ao que tudo indica, envelhecer de maneira bem-sucedida requer um equilibro entre envelhecimento cognitivo e envelhecimento biológico.

A atividade física, por sua vez, mostrou-se como uma grande aliada no retardo das deterioraçóes que acontecem nos sistemas neurológicos e que estão relacionadas à DA. Portanto, a prática regular e sistemática de exercitar o corpo é de extrema importância para a manutenção destes sistemas, evitando ao máximo a evolução para limitaçóes cognitivas e funcionais.

\section{Referências}

BAILEY, D. M. et al. Elevated aerobic fitness sustained throughout the adult lifespan is associated with improved cerebral hemodynamics. Stroke, v. 44, n. 11, p. 3235-3238, 2013. Disponível em: <bit.ly/2KAdbr9>. Acesso em: 13 jun. 2019.

BARNES, D. E.; YAFFE, K. The projected effect of risk factor reduction on Alzheimer's disease prevalence. The Lancet Neurology, v. 10, n. 9, p. 819-828, 2011. Disponível em: <bit. ly/2IGQHCQ>. Acesso em: 13 jun. 2019. 
CAIXETA, L. Doenças de Alzheimer. São Paulo: Artmed Editora, 2009.

CANCELA, J. M. et al. Effects of a long-term aerobic exercise intervention on institutionalized patients with dementia. Journal of science and medicine in sport, v. 19, n. 4, p. 293-298, 2016. Disponível em: <bit.ly/2xePpIs>. Acesso em: 05 jun. 2019.

CARPER, J. 100 dicas simples para prevenir o Alzheimer. São Paulo: Sextante, 2015.

COELHO, F. G. M. de C. et al. Atividade Física e Desempenho Cognitivo em Idosos com Demência de Alzheimer: Uma Revisão Sistemática. Revista Brasileira de Psiquiatria, v. 31, n. 2, 2009. Disponível em: <bit.ly/2X64DyO>. Acesso em: 24 jun. 2019.

DORNELLES, B. Investindo no envelhecimento saudável. Porto Alegre: EDIPUCRS, 2003.

DUZEL, E.; PRAAG, H. V.; SENDTNER, M. Can physical exercise in old age improve memory and hippocampal function? Brain, v. 139, n. 3, p. 662-673, 2016. Disponível em: <bit.ly/2Ry98MK>. Acesso em: 13 jun. 2019.

ERICKSON, K. I.; WEINSTEIN, A. M.; LOPEZ, Oscar L. Physical activity, brain plasticity, and Alzheimer's disease. Archives of medical research, v. 43, n. 8, p. 615-621, 2012. Disponível em: <bit.ly/2xePuvK>. Acesso em: 04 jun. 2019.

FONSECA, V. da. Gerontopsicomotricidade: uma abordagem ao conceito da retrogênese psicomotora. Fonseca V. Psicomotricidade: filogênese, ontogênese e retrogênese. Porto Alegre: Artes Médicas, p. 343-81, 1998.

GARUFFI, M. et al. Atividade física para promoção da saúde de idosos com doença de Alzheimer e seus cuidadores. Revista Brasileira de Atividade Física \& Saúde, v. 16, n. 1, p. 80-83, 2011. Disponível em: <bit.ly/ 2KCfMkx>. Acesso em: 24 jun. 2019.

GUERRA, Y. de S. et al. Ejercicio y enfermedad de Alzheimer: el cuerpo como un todo. Revista Andaluza de Medicina del Deporte, v. 10, n. 3, p. 120-124, 2017. Disponível em: <bit.ly/2YeBvCi $>$. Acesso em: 04 jun. 2019.

HERNANDEZ, S. S. S. et al. Efeitos de um programa de atividade física nas funçôes cognitivas, equilíbrio e risco de quedas em idosos com demência de Alzheimer. Revista Brasileira de Fisioterapia, v. 14, n. 1, 2010. Disponível em: <bit.ly/2XA1wi4>. Acesso em: 24 jun. 2019.

HOSPITAL SÃO CAMILO et al. 5 passos para ter um envelhecimento saudável. 2018. Disponível em: <bit.ly/ 2x9fLf9>. Acesso em: 25 jun. 2019.

KÜSTER, O. C. et al. Cognitive change is more positively associated with an active lifestyle than with training interventions in older adults at risk of dementia: a controlled interventional clinical trial. BMC psychiatry, v. 16, n. 1, p. 315, 2016. Disponível em: <bit.ly/2NpQBnB>. Acesso em: 11 jun. 2019.

LAM, F. M. H. et al. Physical exercise improves strength, balance, mobility, and endurance in people with cognitive impairment and dementia: a systematic review. Journal of physiotherapy, v. 64, n. 1, p. 4-15, 2018. Disponível em: <bit.ly/2Xt3TU1>. Acesso em: 05 jun. 2019. 
LOPES, M. A.; BOTTINO, C. M. C. Prevalência de demência em diversas regióes do mundo: análise dos estudos epidemiológicos de 1994 a 2000. Arq neuropsiquiatr, v. 60, n. 1, p. 61-9, 2002. Disponível em: <bit.ly/2X2W2be>. Acesso em: 06 jun. 2017.

LOURENCO, M. V. et al. Exercise-linked FNDC5/irisin rescues synaptic plasticity and memory defects in Alzheimer's models. Nature medicine, v. 25, n. 1, p. 165, 2019. Disponível em: <bit.ly/2YfiLSR>. Acesso em: 27 jun. 2019.

MARSHALL, M. J.; HUTCHINSON, S. A. A critique of research on the use of activities with persons with Alzheimer's disease: A systematic literature review. Journal of Advanced Nursing, v. 35, n. 4, p. 488-496, 2001. Disponível em: <bit.ly/31PjexD>. Acesso em: 04 jun. 2019.

POIRIER, J.; GAUTHIER, S. Doença de Alzheimer: o guia completo. Belo Horizonte: MG Editores, 2016.

ROLLAND, Y.; KAN, G. A. V.; VELLAS, B. Physical activity and Alzheimer's disease: from prevention to therapeutic perspectives. Journal of the American Medical Directors Association, v. 9, n. 6, p. 390-405, 2008. Disponível em: <bit.ly/2RBBWUK>. Acesso em: 04 jun. 2019.

RYAN, S. M.; NOLAN, Y. M. Neuroinflammation negatively affects adult hippocampal neurogenesis and cognition: can exercise compensate? Neuroscience $\&$ Biobehavioral Reviews, v. 61, p. 121-131, 2016. Disponível em: <bit.ly/2X1pZsc>. Acesso em: 13 jun. 2019.

SHEN, Y.; LI, R. What do we know from clinical trials on exercise and Alzheimer's disease?. Journal of sport and health science, v. 5, n. 4, p. 397-399, 2016. Disponível em: $<$ bit.ly/2ZKSSLm>. Acesso em: 11 jun. 2019.

SOBOL, N. A. et al. Effect of aerobic exercise on physical performance in patients with Alzheimer's disease.Alzheimer's \& Dementia, v. 12, n. 12, p. 1207-1215, 2016. Disponível em: <bit.ly/2XvbgdA>. Acesso em: 05 jun. 2019.

STRÖHLE, A. et al. Drug and exercise treatment of Alzheimer disease and mild cognitive impairment: a systematic review and meta-analysis of effects on cognition in randomized controlled trials. The American Journal of Geriatric Psychiatry, v. 23, n. 12, p. 1234-1249, 2015. Disponível em: <bit.ly/2x9SWrL>. Acesso em: 11 jun. 2019.

SZETO, J. Y. Y.; LEWIS, S. J. G. Current treatment options for Alzheimer's disease and Parkinson's disease dementia. Current neuropharmacology, v. 14, n. 4, p. 326-338, 2016. Disponível em: <bit.ly/2XE7Sxf>. Acesso em: 11 jun. 2019.

VIDONI, E. D. et al. A community-based approach to trials of aerobic exercise in aging and Alzheimer's disease. Contemporary clinical trials, v. 33, n. 6, p. 1105-1116, 2012. Disponível em: <bit.ly/2x9OY2d>. Acesso em: 04 jun. 2019.

YU, F.; VOCK, D. M.; BARCLAY, T. R. Executive function: Responses to aerobic exercise in Alzheimer's disease. Geriatric Nursing, v. 39, n. 2, p. 219-224, 2018. Disponível em: <bit. ly/31Sg3Fd>. Acesso em: 04 jun. 2019. 